\title{
Dosimetric comparison of dose accumulation between rigid registration and deformation registration in intensity-modulated radiation therapy for large volume non-small cell lung cancer
}

\author{
Jianxin Ren ${ }^{1,2}$, Guanzhong Gong ${ }^{2}$, Xinsen Yao $^{2}$, Yong Yin ${ }^{2}$ \\ ${ }^{1}$ School of Physics and Technology, Wuhan University, Wuhan 430072, China; ${ }^{2}$ Department of Radiation Oncology, Shandong Cancer Hospital and \\ Institute, Shandong First Medical University and Shandong Academy of Medical Sciences, Jinan 250117, China \\ Contributions: (I) Conception and design: J Ren, G Gong, Y Yin; (II) Administrative support: Y Yin; (III) Provision of study materials or patients: \\ J Ren, G Gong, Y Yin; (IV) Collection and assembly of data: J Ren, X Yao; (V) Data analysis and interpretation: All authors; (VI) Manuscript writing: \\ All authors; (VII) Final approval of the manuscript: All authors. \\ Correspondence to: Professor Yong Yin, PhD. Department of Radiation Oncology, Shandong Cancer Hospital and Institute, Shandong First Medical \\ University and Shandong Academy of Medical Sciences, Jinan 250117, China. Email: yinyongsd@126.com.
}

Background: To evaluate the cumulative dose to the target volumes and organs at risk (OARs) after replanning during intensity-modulated radiation therapy (IMRT) for large volume non-small cell lung cancer (NSCLC) based on rigid registration and deformation registration technologies.

Methods: Thirty patients with large volume NSCLC who were treated with IMRT were selected, and two four-dimensional computed tomography (4DCT) scans were acquired before radiotherapy and after 20 fractions of radiotherapy. The initial treatment plan $\left(\mathrm{Plan}_{1}\right)$ based on the average density projection CT $\left(\mathrm{CT}_{1 \text {-avg }}\right)$ of the first 4DCT images and the second treatment plan $\left(\mathrm{Plan}_{2}\right)$ based on $\mathrm{CT}_{2 \text {-avg }}$ of the second 4DCT images were calculated. Then, the dose distributions of $\mathrm{Plan}_{2}$ and $\mathrm{Plan}_{1}$ were accumulated based on rigid and deformation registration technologies to obtain $\mathrm{Plan}_{\text {rig }}$ and $\mathrm{Plan}_{\text {def }}$, respectively. Finally, the volume changes of the gross tumor volume (GTV) and OARs between the two CT scans, and the dose-volume parameters among $\mathrm{Plan}_{1}, \mathrm{Plan}_{2}, \mathrm{Plan}_{\text {rig }}$ and $\mathrm{Plan}_{\text {def }}$ were compared.

Results: Compared with those on the first CT, the mean GTV and heart volume on the second CT decreased by $44.2 \%$ and $5.5 \%$, respectively, while the mean volumes of the ipsilateral lung, contralateral lung and total lung increased by $5.2 \%, 6.2 \%$ and $5.8 \%$, respectively. The differences in the above volume parameters between the two CT scans were statistically significant $(\mathrm{P}<0.05)$. Compared with those in $\mathrm{Plan}_{1}$, the $\mathrm{D}_{95}, \mathrm{D}_{98}$ and $\mathrm{V}_{100 \%}$ values of the IGTV (GTV fusion of $10 \mathrm{CT}$ phases) and planning target volume (PTV) in $\mathrm{Plan}_{2}$ did not change significantly $(\mathrm{P}>0.05)$, and those of $\mathrm{Plan}_{\text {rig }}$ and $\mathrm{Plan}_{\text {def }}$ decreased slightly $(\mathrm{P}<0.05)$. The dose-volume parameters of the spinal cord, heart, ipsilateral lung and total lung in $\operatorname{Plan}_{2}, \mathrm{Plan}_{\text {rig }}$ and $\mathrm{Plan}_{\text {def }}$ were significantly lower than those in $\mathrm{Plan}_{1}(\mathrm{P}<0.05)$. Among these parameters, $\mathrm{V}_{30}$ and the mean dose to the heart in $\mathrm{Plan}_{2}, \mathrm{Plan}_{\text {rig }}$ and $\mathrm{Plan}_{\text {def }}$ decreased by 27.3\%, 16.5\%, and $15.3 \%$ and 15.2\%, 6.6\%, and $5.6 \%$ compared to those in $\mathrm{Plan}_{1}$, respectively; $\mathrm{V}_{20}$ and the mean dose to the total lung in $\mathrm{Plan}_{2}, \mathrm{Plan}_{\text {rig }}$

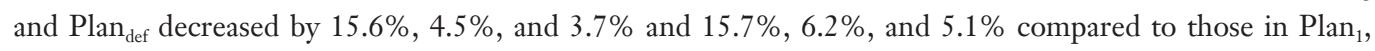
respectively. Some dose-volume parameters (including $\mathrm{D}_{95}$ and $\mathrm{D}_{98}$ to the target volume, $\mathrm{V}_{40}$ of the heart,

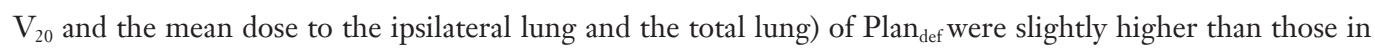
$\mathrm{Plan}_{\text {rig }}(\mathrm{P}<0.05)$. The Dice similarity coefficients (DSCs) of the OARs after deformation registration were significantly higher than those after rigid registration $(\mathrm{P}<0.05)$.

Conclusions: The dose-volume parameters of OARs in $\mathrm{Plan}_{2}$ were noticeably different from those in $\mathrm{Plan}_{1}$, so all of these parameters have large deviations in evaluating the actual dose to the OARs. And, the dose-volume parameters obtained by deformation registration can better predict the actual dose than those obtained by rigid registration.

Keywords: Non-small cell lung cancer (NSCLC); four-dimensional computed tomography (4DCT); deformation 
registration; dosimetric prediction

Submitted Jun 11, 2019. Accepted for publication Oct 10, 2019.

doi: $10.21037 /$ tcr.2019.11.15

View this article at: http://dx.doi.org/10.21037/tcr.2019.11.15

\section{Introduction}

Lung cancer is one of the most common malignant tumors. A worldwide epidemiological survey shows that lung cancer ranks first in the number of new cases and deaths (1). Nonsmall cell lung cancer (NSCLC) accounts for approximately $80 \%$ of lung cancer, mainly including adenocarcinoma and squamous cancer (1). Recent studies have shown that radiotherapy for early NSCLC is almost comparable to surgical treatment, and radiotherapy is also the most important treatment for NSCLC patients who cannot undergo surgery (2).

With the continuous development of radiotherapy technology, intensity-modulated radiation therapy (IMRT) has become one of the most commonly used precision radiotherapy techniques for the treatment of NSCLC (3). The basic assumption of any radiotherapy plan is that the planning target volume (PTV) can reflect the real tumor margin, so the dosimetric profile will be basically consistent with the PTV in the planning design (4). However, during radiotherapy, the real tumor is constantly undergoing changes, such as changes in the motion pattern, progression or regression, and shifts from the baseline position, which may lead to an insufficient dose to the target and leakage of the dose to the organs at risk (OARs). These changes will not only reduce the tumor control rate but also increase the radiation toxicity to the OARs, which is quite dangerous in radiotherapy $(5,6)$. The effect of tumor changes in the motion pattern can be reduced by four-dimensional computed tomography (4DCT) $(7,8)$. However, the effect of tumor regression is difficult to determine, especially for large volume NSCLC, because this type of tumor shrinks significantly during radiotherapy.

At present, to achieve an accurate dose delivery and obtain an accurate dose prediction model, adaptive radiotherapy through daily images of patients is generally necessary (9). However, daily images are difficult to acquire in many institutions, and repositioning and replanning after approximately 20 fractions of radiotherapy is more common $(4,10)$. Moreover, the second round of planning is usually based on a second CT scan, without consideration of the initial treatment plan, which may affect the dosimetric outcomes of the entire treatment (11). Therefore, obtaining the cumulative dose of the whole treatment from multiple plans based on different sets of CT images is very important.

The application of deformable image registration technology in the registration of lung images has been extensively studied in recent years (12). By determining the voxel-to-voxel correspondence between different images, this technology can propagate the planned contour and accumulate the delivered dose. Several studies have shown that the accuracy of lung CT scan registration is very high, and the average error can be less than $1 \mathrm{~mm}$, which provides a basis for the dose accumulation of radiotherapy for NSCLC $(13,14)$.

After replanning, the dose-volume parameters and dosevolume histograms (DVHs) of the two radiotherapy plans cannot accurately predict the actual dose or the incidence of radiation toxicity to the OARs $(15,16)$. Therefore, in this study, we obtained the cumulative dose of the two radiotherapy plans based on rigid registration and deformation registration techniques and compared with the dosimetric parameters of the two plans to provide a more accurate method to reflect the actual dose to the OARs.

\section{Methods}

\section{General data}

Thirty patients with large volume NSCLC who underwent IMRT at the Shandong Cancer Hospital and Institute from September 2016 to August 2018 were enrolled in this study, including 26 males and 4 females, aged $48-81$ years (median 63 years), and the gross tumor volume (GTV) before radiotherapy was $65.1-350.9 \mathrm{~cm}^{3}$ (median $168.6 \mathrm{~cm}^{3}$ ). The inclusion criterion was a GTV greater than $65 \mathrm{~cm}^{3}$.

\section{Posture fixation and CT scanning}

All patients were placed on the treatment bed in the supine position, fixed by a thermoplastic mask or vacuum bag 


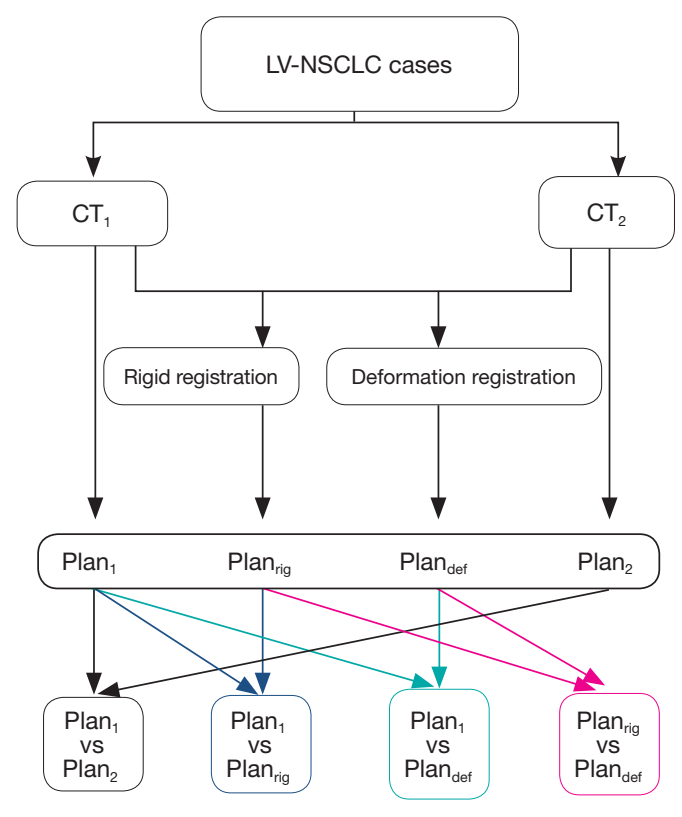

Figure 1 Workflow chart of the dose accumulation process and dosimetric comparison.

and scanned with a Brilliance CT Big Bore device (Philips Healthcare, The Netherlands) to obtain 4DCT scans under quiet breathing. An infrared fluorescence module was placed in the sagittal process of the patient's chest, while an infrared camera acquisition module was placed at the end of the bed to obtain the patient's motion track. The obtained data were transmitted to a real-time position management system (Real-time Position Management, RPM; Varian Medical Systems, USA) to obtain the patient's respiratory signal. The respiratory cycle was divided into 10 phases: $0 \%$, $10 \%, 20 \%, \ldots 90 \%$, of which $50 \%$ was the end-inspiratory phase. The corresponding CT images $\mathrm{CT}_{0}, \mathrm{CT}_{10}, \mathrm{CT}_{20}, \ldots$ $\mathrm{CT}_{90}$, were reconstructed in each phase. The average density projection image $\mathrm{CT}_{\text {avg }}$ was obtained based on the above 10 CTs. Each patient underwent two 4DCT scans before treatment and after 20 fractions of treatment. The scanning thickness and interval were both $3 \mathrm{~mm}$. The scanning range was from $3 \mathrm{~cm}$ above the clavicle to the lower abdomen.

\section{Delineation of the target area and OARs}

Two 4DCT images were introduced into the Eclipse 13.5 three-dimensional treatment planning system (Varian Medical Systems, USA). The GTVs were delineated on the $\mathrm{CT}_{0}, \mathrm{CT}_{10}, \mathrm{CT}_{20}, \ldots \mathrm{CT}_{90}$ images and fused into the IGTV. A $0.8-1.2 \mathrm{~cm}$ extension of the IGTV was considered the
PTV. Then, the normal lung (including the ipsilateral lung and contralateral lung), heart, spinal cord and other OARs were delineated on the CTavg images. The normal lung was lung tissue that excluded the IGTV. After the second scan, the target and OARs were redelineated, and the delineations for each patient were completed by the same clinician.

\section{Planning design}

Then, physicists formulated 5-7 field coplanar IMRT plans in the Eclipse 13.5 planning system. The prescription dose to the PTV was 50-66 Gy (median $60 \mathrm{~Gy}$ ), divided into 2 Gy/every fraction, 5 fractions a week, for a total of 25-33 fractions. The dose limit to the PTV was $95 \%$ volume dose $\left(D_{95}\right)>$ prescription dose, maximum dose $\left(D_{\text {max }}\right)<110 \%$ of prescription dose, and mean PTV dose (MPD) $>102 \%$ of the prescription dose. The dose limit to the spinal cord was a $\mathrm{D}_{\max }<45 \mathrm{~Gy}$. The dose limit to the normal lung was dose volume $\mathrm{V}_{20}<30 \%, \mathrm{~V}_{30}<20 \%$, and mean lung dose (MLD) $<20$ Gy. The dose limit to the heart was $\mathrm{V}_{30}<40 \%$, $\mathrm{V}_{40}<30 \%$, and mean heart dose (MHD) $<25$ Gy. The optimal weight from high to low were $\mathrm{PTV}>$ spinal cord $>$ lung > heart. $\mathrm{Plan}_{1}$ was calculated on $\mathrm{CT}_{1 \text {-avg, }}$ and $\mathrm{Plan}_{2}$ was calculated on $\mathrm{CT}_{2 \text {-avg }}$ after 20 fractions of treatment.

\section{Dose registration accumulation}

The CT images, delineated structures and dose distribution of the two treatment plans were transmitted to Velocity 3.2.1 software (Varian Medical Systems, USA). The dose distribution of the second plan, $\mathrm{Plan}_{2}$ (based on $\mathrm{CT}_{2 \text {-avg }}$ ), was rigidly registered to $\mathrm{CT}_{1 \text {-avg }}$ and then accumulated with the dose distribution of the initial plan, $\mathrm{Plan}_{1}$, to obtain Plan $_{\text {rig }}$ (based on $\mathrm{CT}_{1 \text {-avg }}$ ). Similarly, the dose distribution of $\mathrm{Plan}_{2}$ (based on $\mathrm{CT}_{2 \text {-avg }}$ ) was deformably registered to $\mathrm{CT}_{1 \text {-avg }}$ and then accumulated with the dose distribution of $\mathrm{Plan}_{1}$ to obtain $\mathrm{Plan}_{\text {def }}$ (based on $\mathrm{CT}_{1 \text {-avg }}$ ). The workflow chart of the dose accumulation process is depicted in Figure 1. Dice similarity coefficients (DSCs) were used to evaluate the accuracy of image registration.

\section{Statistical parameters}

The following parameters of $\mathrm{Plan}_{1}, \mathrm{Plan}_{2}, \mathrm{Plan}_{\text {rig }}$ and $\mathrm{Plan}_{\text {def }}$ were calculated: (I) changes in GTV on the $\mathrm{CT}_{1-50}$ and $\mathrm{CT}_{2-50}$ at the end-inspiratory phase; (II) volume changes in the spinal cord, heart, ipsilateral lung, contralateral lung and whole lung on the $\mathrm{CT}_{1 \text {-avg }}$ and $\mathrm{CT}_{2 \text {-avg }}$; (III) DSCs of 
Table 1 Changes in the GTVs and volumes of the OARs between the two CT images (mean \pm SD)

\begin{tabular}{|c|c|c|c|c|c|}
\hline Areas & $\mathrm{CT}_{1}\left(\mathrm{~cm}^{3}\right)$ & $\mathrm{CT}_{2}\left(\mathrm{~cm}^{3}\right)$ & Average change rates (\%) & t value & $P$ value \\
\hline Spinal cord & $26.7 \pm 4.4$ & $26.4 \pm 4.6$ & $-0.9 \pm 3.2$ & 1.447 & 0.159 \\
\hline Heart & $683.5 \pm 105.5$ & $647.9 \pm 138.3$ & $-5.5 \pm 9.9$ & 2.845 & 0.008 \\
\hline Ipsilateral lung & $1,492.7 \pm 495.0$ & $1,580.9 \pm 578.6$ & $5.2 \pm 12.8$ & -2.553 & 0.016 \\
\hline Total lung & $3,246.2 \pm 929.3$ & $3,441.0 \pm 1068.6$ & $5.8 \pm 9.9$ & -3.325 & 0.002 \\
\hline
\end{tabular}

GTV, gross tumor volume; OARs, organs at risk.

Table 2 DSC of the two CT image registration methods (mean \pm SD)

\begin{tabular}{lccc}
\hline Areas & Rig-registration & Def-registration & $t$ value \\
\hline IGTV & $0.59 \pm 0.12$ & $0.67 \pm 0.11$ & -8.087 \\
Spinal cord & $0.81 \pm 0.09$ & $0.89 \pm 0.03$ & -5.567 \\
Heart & $0.88 \pm 0.04$ & $0.94 \pm 0.02$ & -8.502 \\
Ipsilateral lung & $0.89 \pm 0.04$ & $0.95 \pm 0.02$ & 0.000 \\
Contralateral lung & $0.91 \pm 0.03$ & $0.95 \pm 0.02$ & -9.637 \\
Total lung & $0.90 \pm 0.03$ & $0.95 \pm 0.02$ & -12.591 \\
\hline
\end{tabular}

DSC, Dice similarity coefficient; IGTV, GTV fusion of 10 CT phases.

the GTVs and OARs of rigid registration and deformation registration; (IV) $\mathrm{D}_{95}, 98 \%$ volume dose $\left(\mathrm{D}_{98}\right)$ and $100 \%$ prescription dose-volume $\left(\mathrm{V}_{100 \%}\right)$ to the IGTV and PTV; (V) $\mathrm{D}_{\max }$ and $1 \mathrm{~cm}^{3}$ volume dose $\left(\mathrm{D}_{1 \mathrm{~cm} 3}\right)$ to the spinal cord; (VI) $\mathrm{V}_{20}, \mathrm{~V}_{30}, \mathrm{~V}_{40}$ and the MHD (17); and (VII) $\mathrm{V}_{5}, \mathrm{~V}_{10}, \mathrm{~V}_{20}$, $\mathrm{V}_{30}$ and MLD of the ipsilateral lung, contralateral lung and total lung $(18,19)$.

\section{Statistical analysis}

Data were expressed as the mean \pm SD, analyzed by SPSS 20.0 (IBM Company, USA) software and paired $t$-tests. $\mathrm{P}<0.05$ indicated a significant difference.

\section{Results}

\section{Volume changes of the target and OARs}

Compared with those on $\mathrm{CT}_{1}$, the GTVs and heart volumes on $\mathrm{CT}_{2}$ decreased by $44.2 \%$ and $5.5 \%$, respectively, while the volumes of the ipsilateral lung, contralateral lung and total lung increased by $5.2 \%, 6.2 \%$ and $5.8 \%$, respectively, which were statistically significant differences $(\mathrm{P}<0.05)$.
The volume changes of the spinal cord were not significant ( $\mathrm{P}>0.05)$, as shown in Table 1 .

\section{DSCs of rigid and deformation image registration}

The DSC of the GTVs after deformation registration was $0.67 \pm 0.11$, which was higher than that of rigid registration $(0.59 \pm 0.12)$. Compared with the DSC of the spinal cord, heart, ipsilateral lung, contralateral lung and total lung, the parameters of deformation registration $(0.89-0.95)$ were significantly higher than those of rigid registration $(0.81-$ 0.91) $(\mathrm{P}<0.05)$. See Table 2.

\section{Target dosimetric variations}

(I) Compared with those of $\mathrm{Plan}_{1}$, the $\mathrm{D}_{95}, \mathrm{D}_{98}, \mathrm{~V}_{100 \%}$ values of IGTV and PTV in $\mathrm{Plan}_{2}$ did not change significantly $(\mathrm{P}>0.05)$. (II) Compared with those of $\mathrm{Plan}_{1}$, the $\mathrm{D}_{95}, \mathrm{D}_{98}$ and $V_{100 \%}$ values of IGTV and PTV in Plan ${ }_{\text {rig }}$ and Plan $n_{\text {def }}$ significantly decreased to a certain extent (6.3-19.9\%) $(\mathrm{P}<0.05)$. (III) Compared with those of $\mathrm{Plan}_{\text {rig }}$, the $\mathrm{D}_{95}, \mathrm{D}_{98}$ and $\mathrm{V}_{100 \%}$ values of IGTV and PTV in Plandef increased 
Table 3 Target dose parameter statistics (mean \pm SD)

\begin{tabular}{|c|c|c|c|c|c|c|c|c|c|c|c|c|c|}
\hline Target & Parameters & Plan $_{1}$ & $\mathrm{Plan}_{2}$ & Plan $_{\text {rig }}$ & Plan $_{\text {def }}$ & $t_{1}$ value & $P_{1}$ value & $t_{2}$ value & $P_{2}$ value & $t_{3}$ value & $\mathrm{P}_{3}$ value & $t_{4}$ value & $\mathrm{P}_{4}$ value \\
\hline \multirow{2}{*}{ IGTV } & $D_{98}(G y)$ & $60.9 \pm 3.9$ & $60.9 \pm 4.0$ & $53.2 \pm 5.3$ & $55.0 \pm 5.7$ & -0.693 & 0.494 & 6.815 & 0.000 & 5.031 & 0.000 & -2.827 & 0.008 \\
\hline & $V_{100}(\%)$ & $99.9 \pm 0.4$ & $97.1 \pm 15.7$ & $90.2 \pm 10.2$ & $93.0 \pm 9.2$ & 0.966 & 0.342 & 5.161 & 0.000 & 4.047 & 0.000 & -4.160 & 0.000 \\
\hline \multirow[t]{2}{*}{ PTV } & $\mathrm{D}_{95}(\mathrm{~Gy})$ & $59.2 \pm 5.5$ & $58.9 \pm 7.5$ & $49.8 \pm 4.7$ & $51.4 \pm 5.4$ & 0.313 & 0.757 & 9.983 & 0.000 & 7.415 & 0.000 & -3.418 & 0.002 \\
\hline & $V_{100}(\%)$ & $95.9 \pm 5.4$ & $97.1 \pm 1.9$ & $76.5 \pm 12.1$ & $81.3 \pm 11.7$ & -1.301 & 0.203 & 7.871 & 0.000 & 6.222 & 0.000 & -6.054 & 0.000 \\
\hline
\end{tabular}

Note: $t_{1}$ and $P_{1}, t_{2}$ and $P_{2}, t_{3}$ and $P_{3}, t_{4}$ and $P_{4}$ represent the paired $t$-test values of $P_{l a n}$ and $\operatorname{Plan}_{2}, \operatorname{Plan}_{1}$ and Plan $_{\text {rig }}$, Plan 1 and Plan ${ }_{\text {def }}$, and Plan $_{\text {rig }}$ and Plan ${ }_{\text {def }}$, respectively. PTV, planning target volume; IGTV, GTV fusion of 10 CT phases.

slightly $(2.3-6.8 \%)$, and the difference was statistically significant $(\mathrm{P}<0.05)$. See Table 3.

\section{OARs dosimetric change}

(I) The dosimetric parameters of $\mathrm{Plan}_{2}$ were significantly lower than those of $\mathrm{Plan}_{1}$. Among the parameters, Dmax and $\mathrm{D}_{1 \mathrm{~cm} 3}$ to the spinal cord decreased by $5.1 \%$ and $8.6 \%$, respectively. $\mathrm{V}_{20}, \mathrm{~V}_{30}$ and MHD to the heart decreased by $22.8 \%, 27.3 \%$ and $15.2 \%$, respectively. $\mathrm{V}_{5}, \mathrm{~V}_{10}, \mathrm{~V}_{20}, \mathrm{~V}_{30}$, MLD to the ipsilateral lung and total lung decreased by 13.6-23.3\% (mean $17.9 \%$ ) and $12.4-19.5 \%$ (mean $15.3 \%$ ), respectively. $\mathrm{V}_{5}$ to the ipsilateral lung and total lung decreased by $13.6 \%$ and $12.4 \%$, respectively, $\mathrm{V}_{20}$ by $19.0 \%$ and $15.6 \%$, respectively, and MLD by $18.6 \%$ and $15.7 \%$, respectively. These differences were significant $(\mathrm{P}<0.05)$.

(II) The dosimetric parameters of $\mathrm{Plan}_{\text {rig }}$ were lower than those of $\mathrm{Plan}_{1}$. Among the parameters, $\mathrm{D}_{\max }$ and $\mathrm{D}_{1 \mathrm{~cm} 3}$ to the spinal cord decreased by $3.6 \%$ and $3.0 \%$, respectively. $V_{20}$, $\mathrm{V}_{30}, \mathrm{~V}_{40}$ and MHD to the heart decreased by $8.0 \%, 16.5 \%$, $26.7 \%$ and $6.6 \%$, respectively. $V_{10}, V_{20}, V_{30}$, and MLD to the ipsilateral lung and total lung decreased by $2.0-8.8 \%$ (mean $5.4 \%$ ) and $3.4-9.2 \%$ (mean 5.8\%), respectively. $\mathrm{V}_{20}$ to the ipsilateral lung and total lung decreased by $4.3 \%$ and $4.5 \%$, respectively, and the MLD decreased by $6.6 \%$ and $6.2 \%$, respectively. These differences were significant $(\mathrm{P}<0.05)$.

(III) The dosimetric parameters of $\mathrm{Plan}_{\text {def }}$ were lower than those of $\mathrm{Plan}_{1}$. Among the parameters, $\mathrm{D}_{\max }$ and $\mathrm{D}_{1 \mathrm{~cm} 3}$ to the spinal cord decreased by $3.7 \%$ and $2.9 \%$, respectively. $\mathrm{V}_{20}, \mathrm{~V}_{30}, \mathrm{~V}_{40}$ and MHD to the heart decreased by $7.5 \%$, $15.3 \%, 22.4 \%$ and $5.6 \%$, respectively. $\mathrm{V}_{10}, \mathrm{~V}_{20}, \mathrm{~V}_{30}$, and MLD to the ipsilateral lung and total lung decreased by 1.6$7.3 \%$ (mean $4.4 \%$ ) and $2.9-7.9 \%$ (mean $4.9 \%$ ), respectively. $\mathrm{V}_{20}$ of the ipsilateral lung and total lung decreased by $3.5 \%$ and $3.7 \%$, respectively, and the MLD decreased by $3.5 \%$ and $5.1 \%$, respectively. These differences were significant $(\mathrm{P}<0.05)$.

(IV) The dosimetric parameters of $\mathrm{Plan}_{\text {def }}$ were slightly higher than those of $\operatorname{Plan}_{\text {rig }}$. Among the parameters, $V_{40}$ of the heart increased by $9.0 \% . \mathrm{V}_{20}$ and MLD to the ipsilateral lung and total lung increased slightly (less than $1.7 \%$ ). See Table 4.

\section{Discussion}

During radiotherapy for NSCLC, the dose factor is one of the independent prognostic factors that affects the overall survival rate of patients $(9,20)$, so the accurate delivery and prediction of the radiotherapy dose is very important. In this study, the effect of respiratory movement could be eliminated by mapping the radiotherapy target area and OARs based on 4DCT. As radiotherapy progresses, the NSCLC tumor volume will regress, and replanning after approximately 20 minutes of radiotherapy can effectively reduce the dose to the OARs (21). A significant dose distribution difference is typically observed between the second plan and the initial plan, and the difference is often larger in NSCLC tumors with larger volumes. An accurate prediction of the actual dose received by patients has always been one of the important challenges of radiotherapy (11).

In this study, we found that after 20 fractions of radiotherapy for large volume NSCLC, the GTV could regress up to $44.2 \%$, while the volume of the total lungs increased by $5.8 \%$. The dose-volume parameters of the OARs in the second plan were much lower than those in the initial plan, in which the maximum dose to the spinal cord decreased by $5.1 \%$, the MHD decreased by $15.2 \%$, and the MLD to the ipsilateral lung and total lung decreased by $18.6 \%$ and $15.7 \%$, respectively. Dial et al. (21) made similar 
Table 4 Dose parameter statistics of the OARs (mean \pm SD)

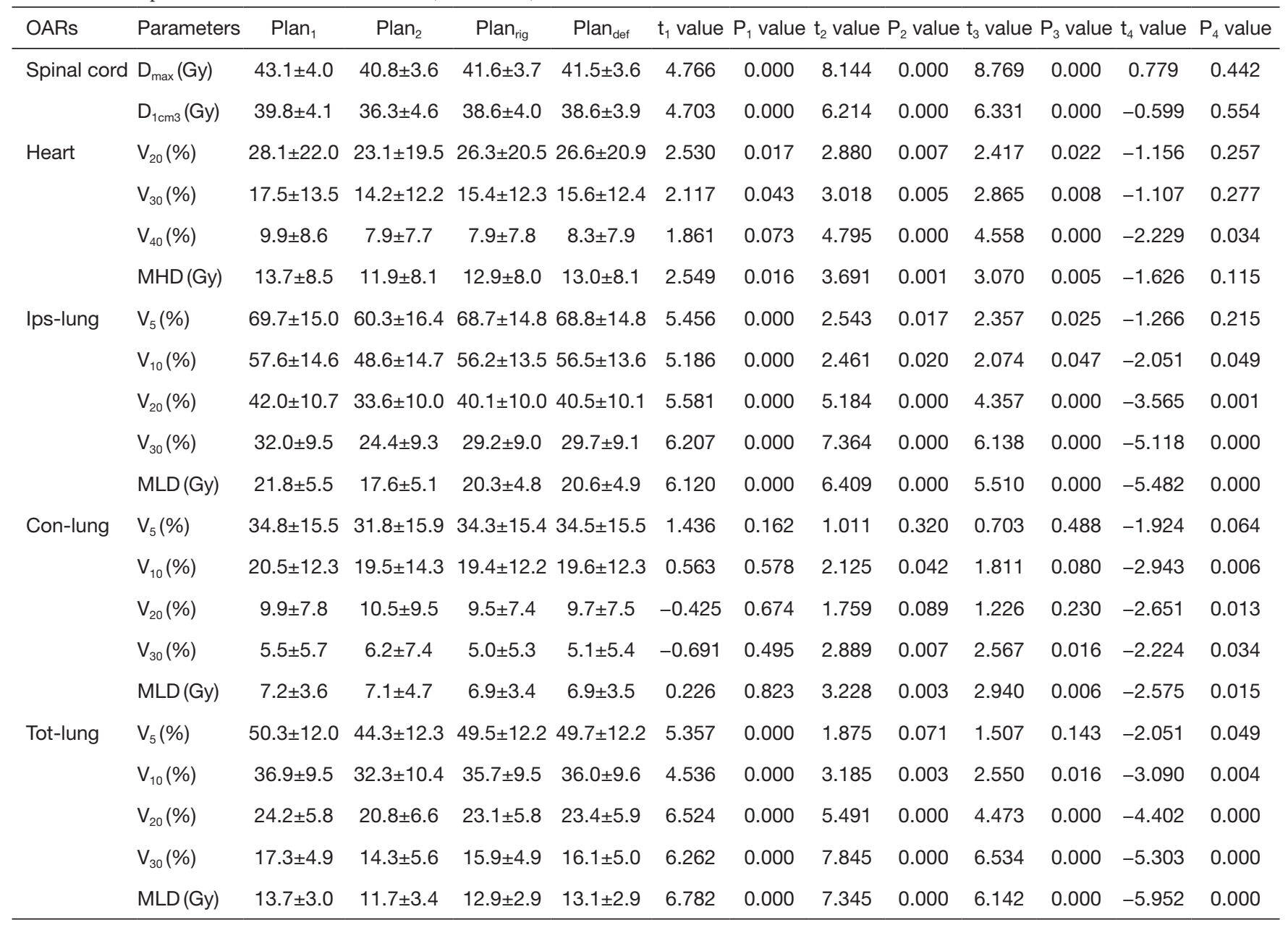

Note: $t_{1}$ and $P_{1}, t_{2}$ and $P_{2}, t_{3}$ and $P_{3}, t_{4}$ and $P_{4}$ represent the paired $t$-test values of Plan $_{1}$ and Plan $_{2}$, Plan and Plan $_{\text {rig }}$, Plan ${ }_{1}$ and Plan ${ }_{\text {def }}$, and

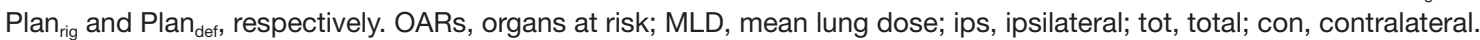

observations after studying 12 patients who underwent adaptive radiotherapy treatment for NACLC. Significant differences exist in the dose-volume parameters of the OARs between the two plans, so using the initial plan or second plan alone to predict the doses to the OARs is not possible.

In this study, the target dose in the cumulative dose was lower than that in the initial plan and the second plan, but this did not mean that the actual target dose was reduced. It was because when we accumulated the doses of the two plans by rigid registration or deformation registration, the dose distribution of the second plan, which had a smaller prescription dose region than the initial plan, was transferred to $\mathrm{CT}_{1}$, which had a larger PTV than $\mathrm{CT}_{2}$, thus the prescription dose region of the second plan could not completely envelope the PTV on $\mathrm{CT}_{1}$. This size difference would underestimate the dose to the target and OARs around the target to a certain extent, and the degree of underestimation with the rigid registration method was greater than that of deformation registration method. Conversely, if the dose distribution of the initial plan, which had a larger prescription dose region than the second plan, was transferred to $\mathrm{CT}_{2}$, which had a smaller PTV than $\mathrm{CT}_{1}$, then the cumulative dose could well reflect the dose to the target area but would to some extent overestimate the dose to the OARs around the target area; the degree of overestimation with the rigid registration method was greater than that with the deformation registration method. This observation indicates that deformation registration is better than rigid registration in the face of large changes in volume and shape. In this study, the dose-volume parameters 
of OARs in Planrig and Plandef were lower than those of the initial plan. Compared to that in the initial plan, the MHD in $\mathrm{Plan}_{\text {rig }}$ and $\mathrm{Plan}_{\text {def }}$ decreased by $6.6 \%$ and $5.6 \%$, and the MLD of the ipsilateral lung decreased by $6.6 \%$ and $5.3 \%$, respectively. Zhong et al. (22) studied 7 patients who underwent adaptive radiotherapy treatment for NSCLC and weekly cone beam computed tomography (CBCT) scans. The rigid and deformed image registration algorithms were used to calculate the dose of each CBCT image and the accumulated dose. Compared with the dose of the initial plan, the MLD of the cumulative dose decreased from 17.3 to 15.2 and 14.5 Gy with the rigid registration and B-spline based deformation registration methods, respectively). The heart volume decreased by $5.5 \%$ on average after 20 fractions of treatment, which was almost the same as that found by Wang et al. (23) and Lutkenhaus et al. (24) and may be associated with changes in myocardial function caused by ionizing radiation that affect cardiac diastolic function and result in a decrease in gross heart volume (25).

In recent years, image deformation registration algorithms based on gray values and feature points have been widely used in the clinic, providing a good tool for dose deformation accumulation in two radiotherapy plans. This study is based on Velocity 3.2.1 software for image registration and dose accumulation. The software uses an improved B-spline algorithm to model the target, which can improve the accuracy of target registration to a clinically acceptable level (26). The DSC values of the IGTV with rigid registration and deformation registration were 0.59 and 0.67 , respectively. Considering that the GTV had a $44.2 \%$ regression, this result is reasonable. The OARs had high DSC values with the rigid registration and deformation registration methods, but the values with the deformation registration method were higher than those with the rigid registration method, in which that of the spinal cord is 0.89 , and that of the heart, ipsilateral lung and contralateral lung were all approximately 0.95 . This observation shows that the accuracy of deformation registration is very high and has little influence on the evaluation parameters.

In conclusion, when treating large volume NSCLC with radiotherapy, predicting the actual dose to the OARs after replanning is a challenging problem. This study found that the cumulative dose based on rigid registration and deformation registration can better reflect the actual dose to the OARs than the initial plan. The cumulative dose calculated by the deformation registration method can better address the volume changes in the target and OARs than that by the rigid registration method, and the deformation registration method had higher DSC values than the rigid registration method. Therefore, we recommend predicting the actual dose to the OARs with the cumulative dose of the two plans obtained by the deformation registration technique to provide a reference for clinical decision-making.

\section{Acknowledgments}

Funding: This research was supported by the Key Research Program of Shandong (No. 2018GSF118006) and the National Key Research and Development Program of China (No. 2017YFC0113202).

\section{Footnote}

Conflicts of Interest: All authors have completed the ICMJE uniform disclosure form (available at http://dx.doi. org/10.21037/tcr.2019.11.15). The authors have no conflicts of interest to declare.

Ethical Statement: The authors are accountable for all aspects of the work in ensuring that questions related to the accuracy or integrity of any part of the work are appropriately investigated and resolved. The study was conducted in accordance with the Declaration of Helsinki (as revised in 2013). This retrospective study was approved by the Institutional Ethics Review Board of the Shandong Cancer Hospital (No. 201809017). Medical record review was performed in accordance with Institutional Ethics Review Board guidelines. Individual informed consent was waived due to the retrospective nature of the study.

Open Access Statement: This is an Open Access article distributed in accordance with the Creative Commons Attribution-NonCommercial-NoDerivs 4.0 International License (CC BY-NC-ND 4.0), which permits the noncommercial replication and distribution of the article with the strict proviso that no changes or edits are made and the original work is properly cited (including links to both the formal publication through the relevant DOI and the license). See: https://creativecommons.org/licenses/by-nc-nd/4.0/.

\section{References}

1. Didkowska J, Wojciechowska U, Mańczuk M, et al. Lung cancer epidemiology: contemporary and future challenges worldwide. Ann Transl Med 2016;4:150.

2. Zhong H, Chetty IJ. Adaptive radiotherapy for NSCLC 
patients: utilizing the principle of energy conservation to evaluate dose mapping operations. Phys Med Biol 2017;62:4333-45.

3. Diwanji TP, Mohindra P, Vyfhuis M, et al. Advances in radiotherapy techniques and delivery for non-small cell lung cancer: benefits of intensity-modulated radiation therapy, proton therapy, and stereotactic body radiation therapy. Transl Lung Cancer Res 2017;6:131-47.

4. Agrawal S, Kumar S, Maurya AK. Potential for adaptive dose escalation in radiotherapy for patients with locally advanced non small cell lung cancer in a low-mid-income setting. Br J Radiol 2017;90:20140234.

5. Liu Z, Su J, Shen W, et al. SU E T 251: analysis of irradiated induced lung injury in non small cell lung cancer (NSCLC) treated by three dimensional conformal radiotherapy (3DCRT). Med Phys 2012;39:3761.

6. Bernchou U, Christiansen RL, Asmussen JT, et al. Extent and computed tomography appearance of early radiation induced lung injury for non-small cell lung cancer. Radiother Oncol 2017;123:93-8.

7. Yuan S, Sun X, Li M, et al. A randomized study of involvedfield irradiation versus elective nodal irradiation in combination with concurrent chemotherapy for inoperable stage III nonsmall cell lung cancer. Am J Clin Oncol 2007;30:239-44.

8. Spoelstra FO, van Sörnsen de Koste JR, Cuijpers JP, et al. Analysis of reproducibility of respiration-triggered gated radiotherapy for lung tumors. Radiother Oncol 2008;87:59-64.

9. Sonke JJ, Belderbos J. Adaptive radiotherapy for lung cancer. Semin Radiat Oncol 2010;20:94-106.

10. Daphtary M, Agrawal S, Vikram B. Human resources for cancer control in Uttar Pradesh, India: a case study for low and middle income countries. Front Oncol 2014;4:237.

11. Xu XM, Deng JJ, Guo HT, et al. CT image fusion in the optimization of replanning during the course of 3-dimensional conformal radiotherapy for non-small-cell lung cancer. 3 rd International Conference on Biomedical Engineering and Informatics 2010;3:1336-9.

12. Muenzing SE, van Ginneken B, Viergever MA, et al. Dirboost-an algorithm for boosting deformable image registration: application to lung CT intra-subject registration. Med Image Anal 2014;18:449-59.

13. Gorbunova V, Sporring J, Lo P, et al. Mass preserving image registration for lung CT. Med Image Anal 2012;16:786-95.

14. Guckenberger M, Baier K, Richter A, et al. Evalution of surface-based deformable image registration for adaptive radiotherapy of non-small cell lung cancer (NSCLC). Radiat Oncol 2009;4:68.

15. Yap ML, Sun A, Higgins J, et al. Adaptive dose escalation using serial four-dimensional positron emission tomography/computed tomography scans during radiotherapy for locally advanced non-small cell lung cancer. Clin Oncol (R Coll Radiol)2016;28:e199-205.

16. Jadon R, Pembroke CA, Hanna CL, et al. A systematic review of organ motion and image-guided strategies in external beam radiotherapy for cervical cancer. Clin Oncol (R Coll Radiol) 2014;26:185-96.

17. Nielsen KM, Offersen BV, Nielsen HM, et al. Short and long term radiation induced cardiovascular disease in patients with cancer. Clin Cardiol 2017;40:255-61.

18. Kim M, Lee J, Ha B, et al. Factors predicting radiation pneumonitis in locally advanced non-small cell lung cancer. Radiat Oncol J 2011;29:181-90.

19. Wang D, Shi J, Liang S, et al. Dose-volume histogram parameters for predicting radiation pneumonitis using receiver operating characteristic curve. Clin Transl Oncol 2013;15:364-9

20. Willner J, Baier K, Caragiani E, et al. Dose, volume, and tumor control prediction in primary radiotherapy of non-small-cell lung cancer. Int J Radiat Oncol Biol Phys 2002;52:382-9.

21. Dial C, Weiss E, Siebers JV, et al. Benefits of adaptive radiation therapy in lung cancer as a function of replanning frequency. Med Phys 2016;43:1787-94.

22. Zhong H, Siddiqui SM, Movsas B, et al. Evaluation of adaptive treatment planning for patients with non-small cell lung cancer. Phys Med Biol 2017;62:4346-60.

23. Wang X, Wang JZ, Li JB, et al. Changes in cardiac volume determined with repeated enhanced 4DCT during chemoradiotherapy for esophageal cancer. Radiat Oncol 2018;13:181.

24. Lutkenhaus LJ, Kamphuis M, van Wieringen N, et al. Reduction in cardiac volume during chemoradiotherapy for patients with esophageal cancer. Radiother Oncol 2013;109:200-3.

25. Hatakenaka M, Yonezawa M, Nonoshita T, et al. Acute cardiac impairment associated with concurrent chemoradiotherapy for esophageal cancer: magnetic resonance evaluation. Int J Radiat Oncol Biol Phys 2012;83:e67-73.

26. Kadoya N, Nakajima Y, Saito M, et al. Multi-institutional validation study of commercially available deformable image registration software for thoracic images. Int J Radiat Oncol Biol Phys 2016;96:422-31.

Cite this article as: Ren J, Gong G, Yao X, Yin Y. Dosimetric comparison of dose accumulation between rigid registration and deformation registration in intensity-modulated radiation therapy for large volume non-small cell lung cancer. Trans Cancer Res 2019;8(8):2878-2885. doi: 10.21037/tcr.2019.11.15 